\title{
MIMO Techniques for High Data Rate Free Space Optical Communication System in Log-Normal Channel
}

\author{
Mohamed R. Abaza*, Raed Mesleh*, Ali Mansour ${ }^{\dagger}$, and Ayman Alfalou ${ }^{\ddagger}$ \\ *Sensor Networks and Cellular System (SNCS) Research Center, University of Tabuk, 71491 Tabuk, Saudi Arabia \\ \{mohamed.raouf.abaza, raed.mesleh\}@ieee.org \\ ${ }^{\dagger}$ Lab STICC, ENSTA Bretagne, 2 Rue François Verny, 29806 Brest Cedex, France, Email: mansour@ieee.org \\ $\ddagger$ Laboratory L@BISEN, ISEN-Brest, CS 42807, 29228 Brest Cedex 2, France, Email: ayman.al-falou@isen.fr
}

\begin{abstract}
Multiple input multiple output (MIMO) technique with equal gain combining (EGC) is considered in this work to enhance the data rate of free-space optical communication (FSO) system in log-normal channel. In FSO system, light propagating in free space is used to transmit data for communication system. 256-ary pulse position modulation (PPM) using intensity modulation with direct detection (IM/DD) as a power efficient system is employed using PIN receiver. Obtained results demonstrate that significant boost of data rate can be achieved using MIMO techniques. Data rate enhances from 350 Mbps to 800 Mbps when using $2 \times 2$ MIMO configuration. As well, 1 Gbps is reported for $4 \times 4$ MIMO system. These enhancements are achieved without any bandwidth or power expansions and the bit error rate (BER) at the receiver is maintained below $10^{-3}$.

Index Terms-Free-space optical communications (FSO), $M$ ary pulse position modulation ( $M$-ary PPM), Multiple input multiple output (MIMO), Log-normal channel, PIN receiver.
\end{abstract}

\section{INTRODUCTION}

Free-space optical (FSO) communication has become more and more interesting over the last two decades as an adjunct or alternative to radio frequency (RF) communication. FSO links involving transmission between two buildings, between ground station and satellite, between end users and fibre optic backbone and as a backup link for optical fibre. Also, FSO is a license-free technology and offers much-enhanced channel bandwidth as compared to RF. Besides, the availability of cheap front-ends makes this technology cost effective when compared to optical-fibre systems [1].

The short wavelengths of the FSO system are easily attenuated by particulate such as fog, haze and rain droplets that are suspended in the air. One of the main problems facing a FSO system is atmospheric turbulence. Optical turbulence arises as a result of random fluctuations in the refractive index of the atmosphere which are directly dependent on fluctuating atmospheric temperature and pressure [2]. The refractive-index fluctuations cause detrimental effects on the optical beam such as beam spreading, irradiance fluctuation and loss of spatial coherence [3, 4$]$.

Pulse position modulation (PPM) using intensity modulation with direct detection (IM/DD) is among the most widely considered modulation technique for FSO system. PPM achieves high power efficiency and improves the system performance at the expense of reduced bandwidth efficiency compared to other modulation schemes such as on-off keying (OOK) [5]. Adopting high order $M$-ary PPM modulation for FSO is shown to enhance the power and bandwidth efficiencies as compared to conventional PPM technique [5,6].

In the past few years, several researchers studied the use of multiple input multiple output (MIMO) concepts in FSO systems. In [7], binary pulse position modulation FSO system in Rayleigh fading channel and with multiple receive units is studied. It is shown that maximal ratio combining (MRC) outperforms other diversity techniques such as selection combining (SC) and equal gain combing (EGC). The concept of coherent optical polarization MIMO scheme has been introduced and discussed in [8].

In this paper, the achieved diversity gain of MIMO system using 256-PPM over log-normal distribution FSO channel for short range communications with IM/DD PIN receiver is investigated. Log-normal distribution is the most widely used model for the probability density function (pdf) of the irradiance because of its accuracy and simplicity [2,9]. MIMO systems utilize the available different channel paths from the different transmit sources to enhance the spectral efficiency and link reliability. In addition, MIMO configurations can be used to achieve high diversity gains to combat channel fading without increasing power or bandwidth $[10,11]$.

The PIN diodes and the avalanche photodiodes (APDs) are the most commonly used photodiodes in FSO. PIN receivers are commonly used due to their low-cost, high mitigation to wide temperature fluctuations and the ability to operate with cheap low-bias voltage power supply. PIN receivers are less sensitive than APD receivers. The sensitivity of these receivers can be reduced by increasing the transmitter power and using larger receiver lens diameter. In case of APD, the increased power margin provides a more robust communication link than PIN receivers. This allows further reduction in transmitter power and the signal to noise ratio (SNR) can be increased through the internal gain of APDs. However, APD receivers are expensive and need high operating voltages which limits their practical usage $[7,12,13]$. 
The remainder of this paper is organized as follows. System model is presented in Section II. Numerical results and discussions are carried out in Section III. Finally, conclusions are presented in Section IV.

\section{FSO MIMO SYSTEM MODEL}

\section{A. Backgrounds Overview}

Short range optical wireless communication links over lognormal channel with variance $\sigma^{2}=0.1$ and zero mean is considered throughout this paper. The pdf of log-normal fading channel is given by [6]

$$
f_{I}(I)=\frac{1}{I \sqrt{8 \pi \sigma^{2}}} \times \exp \left(-\frac{\left(\ln (I)-\ln \left(I_{o}\right)\right)^{2}}{8 \sigma^{2}}\right),
$$

where $f_{I}(I)$ is the pdf of the log-normal channel, $I_{o}$ is the average received signal light intensity without the considered log-normal channel which is depicted in Fig. 1 and $I$ is the received signal light intensity with turbulence. The optical intensity of a source is defined as the optical power emitted per solid angle in units of Watts per Steradian (W/sr) [14].

\section{B. Single Input Single Output (SISO) FSO Link}

FSO SISO system with $M$-ary PPM is considered in [6, 14]. It is shown that the conditional BER in ideal channel condition, i.e., without turbulence, is given by,

$$
\operatorname{Pr}(\mathrm{e} \mid \mathrm{I})=\frac{\mathrm{M}}{2} \times \mathrm{Q}\left(\operatorname{IR}_{\mathrm{p}} \sqrt{\frac{\mathrm{M} \log _{2}(\mathrm{M})}{2 \mathrm{R}_{\mathrm{b}} \sigma_{\text {noise }}^{2}}}\right)
$$

where $Q($.$) is the Gaussian-Q function { }^{1}$ [13], $M$ is the modulation order, $R_{p}$ is the PIN receiver responsivity and $R_{b}$ is the data rate. The noise variance $\left(\sigma_{\text {noise }}^{2}=\sigma_{s h}^{2}+\sigma_{t h}^{2}\right)$ is the summation of the shot noise and the thermal noise variances. The shot noise is caused by background light while the thermal noise is a result of thermally induced random fluctuations in the charge carriers in the resistive element of the photodetector $[6,15]$. The variance of the thermal noise and the variance of the shot noise are given by [6]

$$
\begin{gathered}
\sigma_{t h}^{2}=\frac{4 K_{B} T_{k} B_{e}}{R_{L}} \\
\sigma_{s h}^{2}=2 q_{e} R_{p}\left(I+I_{b}\right) B_{e}
\end{gathered}
$$

with $K_{B}$ being the Boltzmann's constant, $T_{k}$ is the temperature in Kelvin and $B_{e}$ is the equivalent bandwidth of the receiver. The load of the PIN photodetector $\left(R_{L}\right)$ is measured in $(\Omega), q_{e}$ is the electron charge which is measured in Coulombs and $I_{b}$ is the light intensity of the background light.

In this paper, log-normal channel is considered and the BER of $M$-ary PPM for SISO FSO system over log-normal channel is calculated from (2) and given by

$$
\begin{aligned}
& \mathrm{BER}=\frac{\mathrm{M}}{2} \int_{0}^{\infty} \mathrm{Q}\left(\mathrm{IR}_{\mathrm{p}} \sqrt{\frac{\mathrm{M} \log _{2}(\mathrm{M})}{2 \mathrm{R}_{\mathrm{b}} \sigma_{\text {noise }}^{2}}}\right) \mathrm{f}_{\mathrm{I}}(\mathrm{I}) \mathrm{dI} \\
& { }^{1} Q(h)=\frac{1}{\pi} \int_{0}^{\frac{\pi}{2}} \exp \left(-\frac{h^{2}}{2 \sin ^{2} \theta}\right) d \theta
\end{aligned}
$$

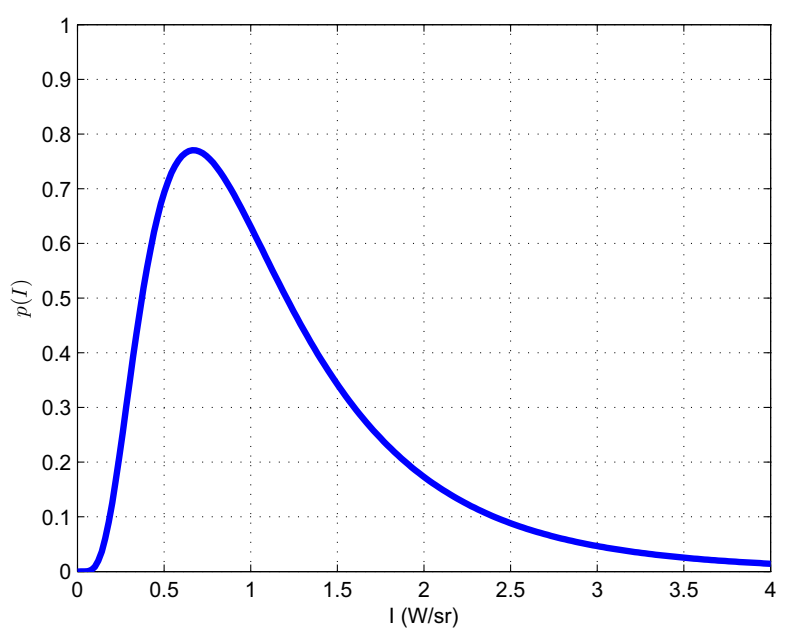

Fig. 1. Log-normal distribution with $\sigma^{2}=0.1$ and with zero mean

Unfortunately, no closed-form solution is available for the integration in (5) and numerical solution is employed in what follows. Solution is obtained through Hermite polynomial. Hermite polynomial approaches the solution through summation of its basis values which are the roots and the weights. The Hermite polynomial formula is given by [16]

$$
\int_{-\infty}^{\infty} g(x) \times e^{-x^{2}} d x \approx \sum_{i=-N, i \neq 0}^{N} w_{i} \times g\left(x_{i}\right)
$$

where $x_{i}$ and $w_{i}$ are the roots and the weights of the Hermite polynomial; respectively and $N$ is the number of summation. It was shown in [13] that accurate results can be obtained if $\mathrm{N} \geq 10$.

Assume $\left(x=\frac{\ln I-\ln I_{o}}{\sqrt{8 \sigma^{2}}}\right)$, the transformation of (5) can be written as

$$
\begin{aligned}
\mathrm{BER}= & \frac{M}{2} \int_{-\infty}^{\infty} e^{-x^{2}} \times \frac{1}{\sqrt{\pi}} \\
& Q\left(I_{o} R_{p} \exp \left(x \sqrt{8 \sigma^{2}}\right) \sqrt{\frac{M \log _{2}(M)}{2 R_{b} \sigma_{\text {noise }}^{2}}}\right) d x
\end{aligned}
$$

Using (6) and (7) the BER can be approximated as

$$
\begin{aligned}
\mathrm{BER} \approx & \frac{M}{2} \sum_{i=-N, i \neq 0}^{N} \frac{1}{\sqrt{\pi}} w_{i} \times \\
& Q\left(I_{o} R_{p} \exp \left(x_{i} \sqrt{8 \sigma^{2}}\right) \sqrt{\frac{M \log _{2}(M)}{2 R_{b} \sigma_{\text {noise }}^{2}}}\right)
\end{aligned}
$$

After a variable change, $I_{i}=I_{0} \exp \left(\sqrt{8 \sigma^{2}} x_{i}\right)$.

$$
\sigma_{s h}^{2}=2 q_{e} R_{p}\left(I_{o} \exp \left(\sqrt{8 \sigma^{2}} x_{i}\right)+I_{b}\right) B_{e}
$$

\section{MIMO FSO Link}

Performance analysis of MIMO FSO system can be derived from its correspondence SISO system. It is shown in [17] 
that the BER expression of MISO FSO system in log-normal channel is similar to that of SISO link with the difference of dividing the variance of the log-normal channel by the number of transmit antennas. Similar conclusion is also drawn in [17] for the case of single input multiple output (SIMO) with EGC. In MIMO system with EGC receiver, however, the variance should be divided by the multiplication of the number transmit and receive units. However, it should be mentioned that these conclusions in [17] are drawn for OOK modulation. This, however, can be generalized to other modulation techniques such as $M$-ary PPM since the assumption depends on the parameters of the log-normal channel and not on the selected modulation technique.

Hence, the BER for a FSO MIMO system in log-normal channel with $A$ transmit and $B$ receive antennas is given by

$$
\begin{aligned}
\mathrm{BER} \approx & \frac{M}{2} \sum_{i=-N, i \neq 0}^{N} \frac{1}{\sqrt{\pi}} w_{i} \times \\
& Q\left(I_{o} R_{p} \exp \left(x_{i} \sqrt{\frac{8 \sigma^{2}}{A \times B}}\right) \sqrt{\frac{M \log _{2}(M)}{2 R_{b} \sigma_{\text {noise }}^{2}}}\right)
\end{aligned}
$$

and the shot-noise variance can be written as

$$
\sigma_{s h}^{2}=2 q_{e} R_{p}\left(I_{o} \exp \left(\sqrt{\frac{8 \sigma^{2}}{A \times B}} x_{i}\right)+I_{b}\right) B_{e}
$$

The synoptic diagram of the proposed model is depicted in Fig. 2. The source bits is modulated using 256-PPM and encoded by the MIMO encoder. The encoded real symbols modulate the laser diodes and the diodes are assumed to be synchronized to start emission simultaneously. The transmitted light propagates over log-normal channel and AWGN due to shot noise and thermal noise are added at the receiver input. At the receiver, the PIN photodiode converts the light intensity into electrical current and EGC is applied to estimate the transmitted information bits. It is assumed that full channel state information is available at the receiver.

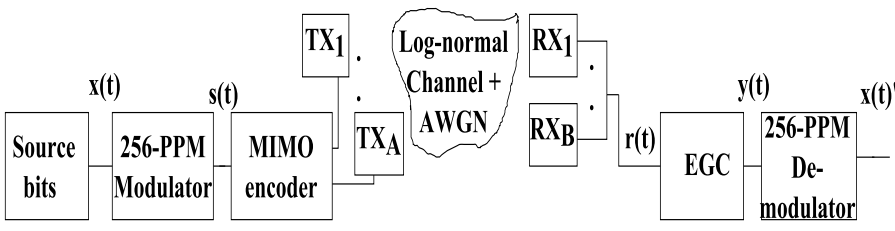

Fig. 2. Synoptic diagram of the proposed model.

\section{NUMERICAL RESULTS AND DISCUSSIONS}

In all conducted analysis, it is assumed that the transmit and receive units are separated wide enough to avoid any correlation among them. Also, a target BER $<10^{-3}$ is considered as a benchmark. It is shown in [18] that an error-free transmission can be achieved when forward error correction (FEC) coding technique is employed at uncoded BER that is less than $10^{-3}$.
The distance between the transmitters and the receivers is set to 100 meters. The electrical bandwidth of the $M$-ary PPM is given by [19]

$$
B W_{\text {electrical }}=\frac{M}{\log _{2}(M)} R_{b}
$$

where $R_{p}=0.9(\mathrm{~A} / \mathrm{W})$ is considered in the following analysis.

In Fig. 3, a background noise of $0 \mathrm{dBm}$ is assumed at a data rate of $350 \mathrm{Mbps}$. In the analysis, the average received light intensity is kept less than $20 \mathrm{dBm}$ which is equivalent to $100 \mathrm{~mW}$. It can be clearly noticed from Fig. 3 that an improvements of $3.77 \mathrm{~dB}$ and $5 \mathrm{~dB}$ can be achieved with $2 \times 2$ and $4 \times 4$ MIMO configurations; respectively. Monte Carlo simulation results are depicted and used to validate the analytical derivations of the BER given in (8).

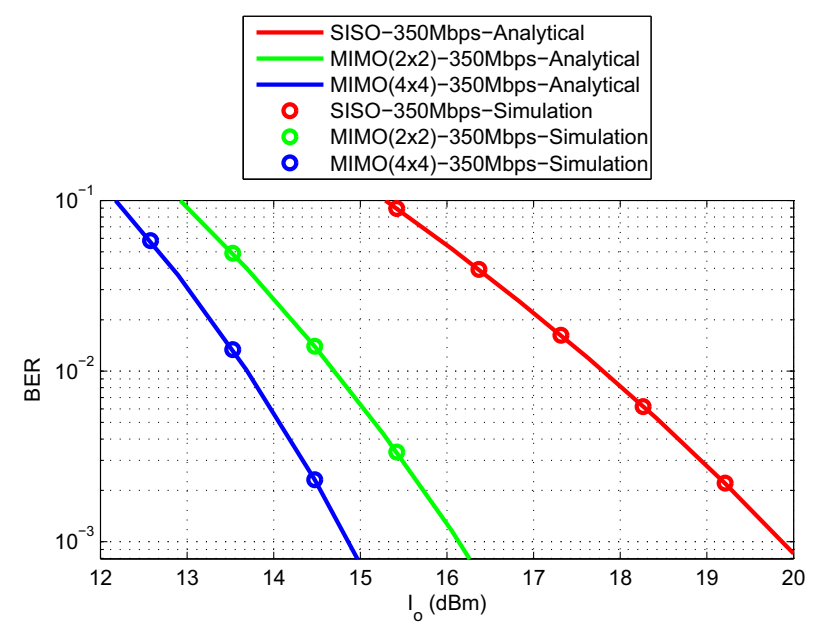

Fig. 3. 256-PPM in log-normal channel using PIN at background noise (0 $\mathrm{dBm}$ ) for the same data rate.

In Fig. 4, the achieved BER for different data rate systems using $2 \times 2$ and $4 \times 4$ MIMO configurations are depicted. Results show that in case of MIMO $(2 \times 2)$ the date rate increased from $350 \mathrm{Mbps}$ in the SISO case to $800 \mathrm{Mbps}$ which is $128 \%$ higher. Also, $0.38 \mathrm{~dB}$ improvement in received light intensity can be noticed at the target BER. In case of MIMO ' $(4 \times 4)$ configuration, the data rate increases by $185 \%$ as compared to SISO and the received light intensity improves by $0.75 \mathrm{~dB}$. Analytical results are depicted to sustain numerical ones.

The effect of varying temperatures on the performance of the considered system is depicted in Fig. 5. Increasing the temperature degrades the overall system performance. This is not limited to MIMO $(2 \times 2)$ but also extended to SISO and MIMO $(4 \times 4)$.

Different PIN responsivity $0.7(A / W)$ and $0.9(A / W)$ are employed for MIMO $(2 \times 2)$ in Fig. 6. The result shows that increasing the value of responsivity improves the BER performance. Using PIN photodetector with $R_{p}=0.9$ enhances the received light intensity by $1 \mathrm{~dB}$. The same effect applies on SISO and MIMO $(4 \times 4)$. 


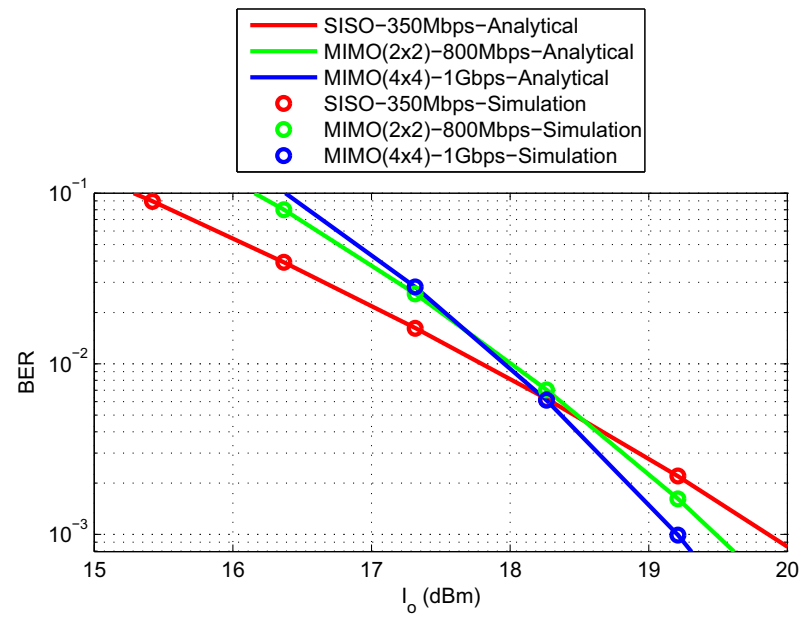

Fig. 4. 256-PPM in log-normal channel using PIN at background noise (0 $\mathrm{dBm})$ for different data rate.

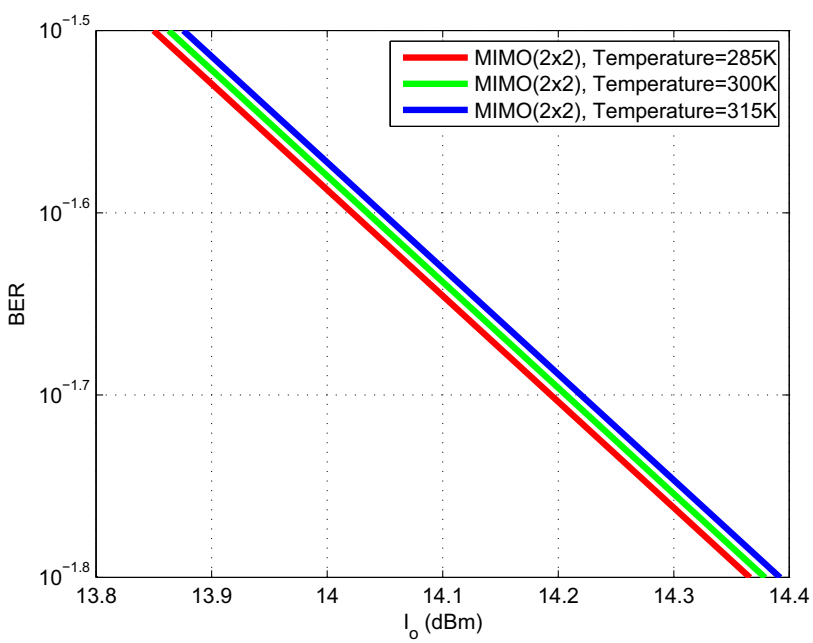

Fig. 5. BER against average received light intensity for different temperatures at $R_{b}=350$ Mbps.

In Figs. $3-6, R_{p}$ is equal to 0.9 Amperes per Watt $(A / W)$ except in Fig. 6. The operating temperature is $300 \mathrm{~K}$ (room temperature) except in Fig. 5. The receiver resistance load is $50 \Omega$. Also, an electrical bandwidth of $11.2 \mathrm{GHz}$ is assumed at the receiver.

From (3), it can be stated that $R_{L}$ is inversely proportional to the variance of the thermal noise which is part of the variance of the total noise. In addition, the total-noise variance is inversely proportional to the BER. Therefore, $R_{L}$ is inversely proportional to the BER.

\section{COnclusions}

MIMO with EGC can be employed to increase the data rate of FSO communications in log-normal channel. 256-ary PPM using IM/DD as a power efficient system is employed using PIN receiver. Our analytical and simulations results show that

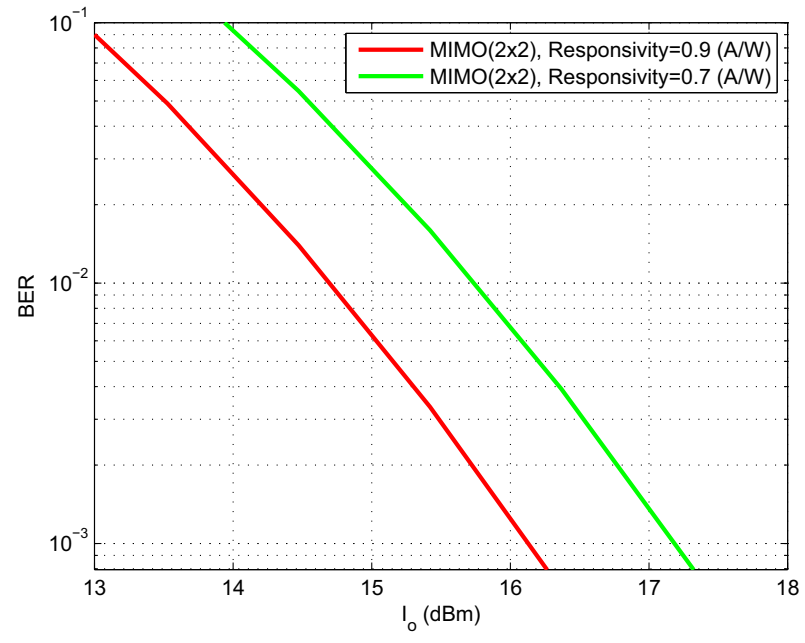

Fig. 6. BER against average received light intensity for different responsivity values at $R_{b}=350 \mathrm{Mbps}$.

the data rate is increased by $128 \%$ and $185 \%$ in case of MIMO $(2 \times 2)$ and MIMO $(4 \times 4)$, respectively compared to SISO without increasing the bandwidth and with less received light intensity. The impact of varying the temperature and the responsivity of the PIN receiver on the overall system performance is investigated as well.

\section{ACKNOWLEDGMENT}

The authors gratefully acknowledge the support for this work from SNCS research center at University of Tabuk under the grant from the Ministry of Higher Education in Saudi Arabia.

\section{REFERENCES}

[1] S. Das, H. Henniger, B. Epple, C. Moore, W. Rabinovich, R. Sova, and D. Young, "Requirements and challenges for tactical free-space lasercomm," in IEEE Military Communications Conference, San Diego, USA, Nov. 17-19, 2008, pp. 1-10.

[2] M. Uysal, J. T. Li, and M. Yu, "Error rate performance analysis of coded free-space optical links over gamma-gamma atmospheric turbulence channels," IEEE Trans. Wireless Commun., vol. 5, no. 6, pp. 1229-1233, Jun. 2006.

[3] A. Aladeloba, A. Phillips, and M. Woolfson, "Performance evaluation of optically preamplified digital pulse position modulation turbulent freespace optical communication systems," IET Optoelectronic, vol. 6, no. 1, pp. 66-74, Feb. 2012.

[4] G. Yang, M.-A. Khalighi, S. Bourennane, and Z. Ghassemlooy, "Approximation to the sum of two correlated gamma-gamma variates and its applications in free-space optical communications," IEEE Wireless Communications Letters, vol. 1, no. 6, pp. 621-624, Dec. 2012.

[5] M. R. Abaza, N. A. Mohammed, and M. H. Aly, "BER performance of M-ary PPM free-space optical communications with channel fading," in Proceeding of the 8th International Conference on High Capacity Optical Networks \& Enabling Technologies (HONET 2011), Riyadh, Saudi Arabia, Dec. 19-21, 2011, pp. 111-115.

[6] J. Yan, Z. Zheng, W. Hu, and A. Xu, "Improved performance of Mary PPM free-space optical communication systems in atmospheric turbulence due to forward error correction," in Proceeding of the 10th International Conference on Commununication Technology (ICCT), Guilin, China, Nov. 27-30, 2006, pp. 1-4. 
[7] H. Shaban, S. D. A. E. Aziz, and M. H. Aly, "Probability of error performance of free space optical systems in severe atmospheric turbulence channels," in 10th Mediterranean Microwave Symposium (MMS'2010), Morphou, Cyprus, Aug. 25-27, 2010, pp. 355-359.

[8] Y. Han and G. Li, "Coherent optical communication using polarization multiple-input-multiple-output," Opt. Express, vol. 13, no. 19, pp. 75277534, Sep. 2005.

[9] N. A. Mohammed, M. R. Abaza, and M. H. Aly, "Improved performance of M-ary PPM in different free-space optical channels due to reed solomon code using APD," International Journal of Scientific \& Engineering Research, vol. 2, no. 4, pp. 82-85, Apr. 2011.

[10] G. Abbas, E. Ahmed, W. Aziz, S. Saleem, and Q. ul Islam, "Performance enhancement of multi-input multi-output (MIMO) system with diversity," International Journal of Multidisciplinary Sciences and Engineering, vol. 3, no. 5, pp. 8-12, May 2012.

[11] R. Mesleh, R. Mehmood, H. Elgala, and H. Haas, "Indoor MIMO optical wireless communication using spatial modulation," in IEEE International Conference on Communications (ICC'10), Cape Town, South Africa, May 22-27, 2010, pp. 1-5.

[12] C. Singh, J. John, Y. N. Singh, and K. K. Tripathi, "A review of indoor optical wireless systems," IETE Technical Review, vol. 19, no. 1-2, pp. 3-17, Jan.-Apr. 2002
[13] K. Kiasaleh, "Performance of APD-based, PPM free-space optical communication systems in atmospheric turbulence," IEEE Transactions on Communications, vol. 53, no. 9, pp. 1455-1461, Sep. 2005.

[14] S. Hranilovic, Wireless Optical Communication Systems, 1st ed. Springer, Sep. 1996, pp. 10-55.

[15] M. L. Baedke, "Performance analysis of multipulse PPM on MIMO free-space optical channels," Ph.D. dissertation, School of Engineering and Applied Science, University of Virginia, Virginia, USA, Aug. 2004

[16] M. Abramowitz and I. A. Stegun, Handbook of Mathematical Functions with Fomulas, Graphs, and Mathematical Tables, 9th ed. Dover Publications, 1972.

[17] S. M. Navidpour, M. Uysal, and M. Kavehrad, "BER performance of free-space optical transmission with spatial diversity," IEEE Trans. Wireless Commun., vol. 6, no. 8, pp. 2813-2819, Aug. 2007.

[18] Z. Wang, W.-D. Zhong, S. Fu, and C. Lin, "Performance comparison of different modulation formats over free-space optical (FSO) turbulence links with space diversity reception technique," IEEE Photonics Journal, vol. 1, no. 6, pp. 277-285, Dec. 2009.

[19] N. Chand, A. J. Hunton, and B. M. Eteson, "A comparative study of $2.667 \mathrm{~Gb} / \mathrm{s}$ OOK, DPSK, and PPM modulation formats for FSO applications," Proceeding of the SPIE, vol. 7091, pp. 1-7, Aug. 2008. 